\title{
MÉXICO Y LA SEGURIDAD ALIMENTARIA: EL USO DE LAS TIC PARA UN ETIQUETADO DIGITAL EN ALIMENTOS
}

\author{
MEXICO AND FOOD SAFETY: ICT \\ FOR DIGITAL FOOD LABELING*
}

\section{Paulina Elisa LAGUNES NAVARRO**}

RESUMEN: El problema multifactorial que representa la lucha contra la obesidad y la diabetes en México, se centra en dos factores principales: el primero se aboca en el etiquetado, cuyo objetivo es informar al consumidor sobre el contenido del alimento y su aporte nutricional, sin embargo, tal objetivo se ve obstruido al no manejar un código en común que el consumidor pueda comprender; mientras el segundo factor, involucra la ausencia de educación nutricional del consumidor para cambiar hábitos alimenticios.

Esta situación conlleva a plantear la innovación del etiquetado por medio de las Tecnologías de la Información y Comunicación (TIC) considerando al código QR y a la realidad aumentada como aliados para atender la necesidad de hacer completa, exacta y entendible la información del etiquetado para el consumidor y fomentar la educación nutricional en éste.

Palabras clave: etiquetado en alimentos, TIC, seguridad alimentaria, derechos del consumidor, código QR, realidad aumentada, educación nutricional, inclusión.
ABSTRACT: The multifaceted problem of the fight against obesity and diabetes in México, revolves around two main factors. The first one focuses on food labelling, which aims to inform consumers about the nutritional content and information. However, this objective es hampered by not having a standardized code that consumers can understand. The second factor is the absence of food education for consumers to change their eating habits.

This situation raises the question of innovating these labels through the use of Information and Communication Technologies (ICT) with QR Codes and augmented reality as allies to make labelling information more complete, precise and understandable for consumers, and to contribute to their nutritional education.

Keyzords: food labelling: ICT, food safety, consumer rights, QR Code, Augmented Reality, nutrition education, social inclusion.

* Recibido el 25 de febrero de 2020; aprobado el 15 de septiembre de 2020.

** ORCID: 0000-0003-3259-7772. Investigadora en el INFOTEC, Centro de Investigación e Innovación en Tecnologías de la Información y Comunicación. Correo electrónicopaulina.lagunesn@gmail.com

Boletín Mexicano de Derecho Comparado, nueva serie, año LIII, núm. 158, mayo-agosto de 2020, pp. 763-787.

Esta obra está bajo una Licencia Creative Commons Atribución-NoComercial-SinDerivar 4.0 Internacional, IIJ-UNAM. 
SUMARIO: I. Introducción. II. El contexto mexicano en materia de etiquetado en alimentos. III. El uso de las TIC en el etiquetado de alimentos. IV. Los obstáculos del etiquetado digital. V. Conclusión. VI. Bibliografia.

\section{INTRODUGGIÓN}

Desde pequeños nos enseñan la importancia de poder comunicarnos con los demás. Para ello, es necesario que existan dos partes: el emisor y el receptor, en donde el primero, es quien emite el mensaje; mientras el segundo, es quien lo recibe y descifra; de esta forma, las personas acceden a la información proporcionada por otras (Araya 2017, 32-34). Sin embargo, para que la comunicación sea óptima se requiere que el mensaje esté bajo un código en común (Araya 2017, 32-34).

Tal acto rutinario pareciera de fácil realización para cualquier persona, pero no para las personas morales; es decir, a partir de que se reconoció el derecho al acceso a la información, el 11 de junio de 2013, se ha debatido sobre los límites de este derecho en cualquier materia, como es el caso del etiquetado de los productos alimenticios. Este derecho se encuentra reconocido en el artículo 6o., párrafo segundo, de la Constitución Política de los Estados Unidos Mexicano (en adelante, CPEUM).

El etiquetado es un instrumento que se encuentra relacionado con la figura de la seguridad alimentaria, la cual fue definida en 1996 en la Cumbre Mundial de la Alimentación, como: “[...] cuando todas las personas, en todo momento, tienen acceso físico y económico a suficiente alimento, seguro y nutritivo, para satisfacer sus necesidades alimenticias y sus preferencias, con el objeto de llevar una vida activa y sana" (FAO-Programa Especial para la Seguridad Alimentaria (PESA) - Centroamérica 2011, 2, en adelante, FAO-PESA Centroamérica).

De dicha definición derivan cuatro dimensiones que garantizan la seguridad alimentaria: 1) disponibilidad; 2) estabilidad; 3) acceso económico y físico a los alimentos, y 4) consumo y utilización biológica de los alimentos. Es decir, un país debe de tener alimento suficiente y accesible para todos sus gobernados durante todo el año sin importar las circunstancias; asimismo, estos alimentos deben de aportar un valor nutricional adecuado, suficiente e inocuo para su ingesta con la finalidad de salvaguardar la salud del consumidor (FAO-PESA Centroamérica 2011, 4; FAO 2011, 1). Para efectos de este trabajo, nos centraremos en la cuarta dimensión, es 
decir, el consumo y utilización biológica de los alimentos ya que se relaciona con el aporte nutricional de estos (cfr. FAO 2011, 1).

El etiquetado involucra a dos derechos sociales: el derecho a la salud y el derecho a la alimentación, ambos consagrados en los artículos 11 y 12 del Pacto Internacional de los Derechos Económicos Sociales y Culturales (en adelante PIDESC), entre otros ordenamientos jurídicos.

De esta forma, el etiquetado contribuye en la promoción y protección de ambos derechos sociales, dado que es el medio de comunicación entre las empresas y los consumidores, en donde se le informa a este último sobre el contenido del producto alimenticio que adquiere y consume; así como el valor nutricional, los ingredientes que lo conforman (incluyendo los componentes alergénicos), la forma de preparación del producto y la proporción de datos sobre el valor calórico, azúcares, carbohidratos, grasas, entre otros (cfr. Stern et al. 2011, 9).

Esta información, concientiza e influye en las decisiones del consumidor sobre el valor nutricional del producto y si cumple con los estándares establecidos para llevar una dieta balanceada, sana, nutritiva y de calidad (cfr. Cowburn y Stockley 2005, 22); conforme a lo establecido en el artículo 4o., tercer párrafo de la CPEUM.

No obstante, en un estudio realizado por Stern, Dalia et al., titulado Revisión del etiquetado frontal: análisis de las guias diarias de alimentación (GDA) y su comprensión por estudiantes de nutrición en México, señalaron que existen dos problemáticas: 1) la falta de comprensión del etiquetado frontal y 2) la escasa educación nutricional de los consumidores (cfr. Stern et al. 2011, 19-20 y 25-34). Es decir, las empresas productoras de alimentos transmiten un mensaje cuyo código no es común para los consumidores, lo cual provoca que no cumpla con su objetivo de informar.

Esta situación vulnera algunos de los principios establecidos en el párrafo 5o. de las Directrices de la Naciones Unidas para la Protección del Consumidor, las cuales son:

[...] b) La protección de los consumidores en situación vulnerable y de desventaja; c) La protección de los consumidores frente a los riesgos para su salud y su seguridad; d) La promoción y protección de los intereses económicos de los consumidores; e) El acceso de los consumidores a una información adecuada que les permita hacer elecciones bien fundadas conforme a los deseos y necesidades de cada cual; f) La educación del consumidor, incluida 
la educación sobre las consecuencias ambientales, sociales y económicas que tienen sus elecciones; $[. .$.

Asimismo, el artículo 1o. de la Ley Federal de Protección al Consumidor señala que dentro de sus principios se encuentra la protección de la vida, salud y seguridad del consumidor en contra de las prácticas engañosas, productos dañinos para su salud, acceso a información clara y adecuada en el etiquetado para la toma de decisión del consumidor, para ello es necesario la educación y divulgación sobre adquisición de los productos alimenticios; de igual forma, se debe proteger los derechos de los grupos vulnerables como: niñas, niños y adolescentes; adultos mayores; personas con discapacidad e indígenas. ${ }^{1}$

Cabe mencionar que México tiene un problema de salud pública al ser uno de los países con mayor índice de obesidad y diabetes en el mundo, lo cual ha ocasionado que se adopten diversas medidas desde los impuestos sobre alimentos y bebidas hasta las reformas, adiciones y modificaciones a la Ley General de Salud (en adelante LGS) y las Normas Oficiales Mexicanas (NOM) (cfr. FAO 2019; Instituto Nacional de Salud Pública y UNICEF México 2016, y Montes de Oca Barrera 2019, 13-14 y 55-57).

Aunado a lo anterior, la Organización Panamericana de la Salud (OPS) y la Organización Mundial de la Salud (OMS) elaboraron la "Estrategia para la prevención y el control de las enfermedades no transmisibles, 2012-2025", algunos de sus objetivos son: el prevenir y controlar las enfermedades crónicas no transmisibles (en adelante ENT) y sus factores de riesgo (OPS-OMS 2012).

En consecuencia, la región de América Latina ha modificado la normativa en materia de etiquetado en países como Chile, Perú, Uruguay y México, con la finalidad de establecer un etiquetado frontal de advertencia (EFA) que apoye al consumidor a comprender el significado del valor nutricional de los alimentos que consume. Sin embargo, se considera que tal avance debe ir acompañado de la innovación mediante el uso de las Tecnologías de la Información y Comunicación (TIC) que contribuiría a atender la falta de educación nutricional.

1 Publicado en el Diario Oficial de la Federación, México, 24 de diciembre de 1992. Última reforma publicada el 12 de abril de 2019. 
Dicho lo anterior, este trabajo se divide en tres secciones: en el primero, se describe el contexto del Estado mexicano en materia de alimentación y etiquetado; en el segundo, se enfoca en cómo emplear las TIC para mejorar el medio de comunicación entre el productor y el consumidor, y en la tercera sección, se aboca en los obstáculos del uso de las TIC en materia de etiquetado digital en México.

\section{EL GONTEXTO MEXICANO EN MATERIA DE ETIQUETADO EN ALIMENTOS}

En México, las reformas constitucionales del 6 y 10 de junio de 2011 en materia de derechos humanos y amparo trajeron consigo nuevas figuras jurídicas al derecho mexicano, puesto que los artículos lo. y 133 de la CPEUM son los ejes rectores para lograr la apertura a la exigibilidad y justiciabilidad de los derechos sociales, tales como la alimentación y la salud, mediante dos principios: dignidad humana y pro persona, los cuales brindan una mayor protección jurídica a la persona en materia de derechos humanos al considerar no sólo el derecho interno sino también a los tratados internacionales de los cuales México es parte (Suprema Corte de Justicia de la Nacíon (SGJN) 2011).

Según Baxi Upendra, los derechos sociales derivan de la realidad y la búsqueda de justicia ante los abusos a los derechos de las personas ( $c f r$. Upendra 2007, 11 y 12). Aunque, Paz González afirma que un efecto adverso que tuvo el Estado del bienestar fue privilegiar al interés económico sobre los demás intereses, lo cual fue un derivado de la inexistencia normativa para modular al sector privado y derivó en abusos que superaron al Estado (Paz González 2017, 5-6 y 13).

No obstante, en un Estado constitucional, los derechos sociales tienen un rol relevante pues ellos conllevan a la inclusión, lo cual favorece la democracia y la participación social; de esta forma, se procura que los derechos humanos sean una necesidad y no se considere como un lujo (Mazhar 2011, 304 y 305). Por su parte, Ferrajoli señala que al garantizar los derechos sociales se procura el desarrollo económico, pues de esto depende el mejorar la calidad de vida de la sociedad (cfr. Ferrajoli 2011, 107 y 108). 
Ahora bien, la CPEUM, en su artículo 1o., párrafo tercero, invoca la obligación que tienen las autoridades de promover, respetar, proteger y garantizar los derechos humanos, con la finalidad de salvaguardar la dignidad humana de las personas. ${ }^{2}$ Adicionalmente, el artículo 25, primer párrafo, de la CPEUM señala que el desarrollo nacional debe ser integral y sustentable para fortalecer la soberanía nacional, el comercio y garantizar la dignidad humana y mejorar la calidad de vida de las personas.

Es ahí en donde la sustentabilidad y el principio de dignidad humana nos conlleva a que el Estado tiene la obligación de vigilar que dentro de su territorio o jurisdicción las empresas sigan los principios rectores que estén encaminados a proteger y garantizar los derechos humanos; por ende, el Estado debe estar al pendiente de las empresas y del impacto de sus actividades que éstas generen en los derechos humanos de los ciudadanos del país, conforme lo estipulado en las Directrices de la Naciones Unidas para la Protección del Consumidor y a la Ley Federal de Protección al Consumidor, anteriormente mencionados (Naciones Unidas 2011, 3 y 5).

Dicho lo anterior, una parte de la base del bienestar social se encuentra en la salud, la cual está ampliamente relacionada a la alimentación nutritiva y de calidad; es decir, en el ámbito internacional, el derecho a la salud se define como "[... el] disfrute del más alto nivel posible de salud física y mental [... incluso social]"; por lo que debe considerar las medidas para prevenir o tratar enfermedades como las ENT (verbigracia sobrepeso, obesidad, diabetes), según el artículo 12, puntos 1 y 2 en su inciso c, del PIDESC, y 10 punto 1 y 2 incisos d y e del Protocolo Adicional a la Convención Americana sobre Derechos Humanos en materia de Derechos Económicos, Sociales y Culturales: "Protocolo de San Salvador", así como la Observación General No. 14, "El derecho al disfrute del más alto nivel posible de salud" (en adelante Observación General No. 14).

Bajo el mismo tenor, el derecho a la alimentación puede ser llevado a ciertos extremos ya sea a la malnutrición, desnutrición o al sobrepeso,

2 Cfr. rubro: Dignidad humana. Constituye un derecho fundamental que es la base de los demás derechos humanos reconocidos constitucional y convencionalmente (SCJN 2018, Tesis: I.10o.A.1 CS (10a.)); rubro: DignidAD HUMANA. SU NATURALEZA Y CONCEPTO (SCJN 2011, Tesis: I.5o.C. J/31 (9a.)); rubro: DignidaD humana. Definiaión. (SCJN 2011, Tesis: I.5o.C. J/30 (9a.)), y rubro: DignidAd HUMANA. ConstituYe UnA NORMA JURÍDICA QUE CONSAGRA UN DERECHO FUNDAMENTAL A FAVOR DE LAS PERSONAS Y NO UNA SIMPLE DECLARACIÓN ÉTICA (SCJN, 2016, Tesis: 1a./J. 37/2016 (10a.)). 
obesidad y/o diabetes, entre otras, tal derecho se encuentra estipulado en los artículos 11 del PIDESC; 4.1 de la Convención Americana sobre Derechos Humanos, OEA; 25, párrafo primero, de la Declaración Universal de los Derechos Humanos.

Adicionalmente, el Protocolo de San Salvador define a este derecho como "[...] una nutrición adecuada que le asegure la posibilidad de gozar del más alto nivel de desarrollo físico, emocional e intelectual”, según el artículo 12 de dicho ordenamiento jurídico. Así mismo, la Observación General No. 14 menciona en párrafo 43, inciso b, las características al derecho a la alimentación como nutritiva, adecuadas y segura.

En cuanto a la legislación mexicana, los derechos a la salud y a la alimentación nutritiva, suficiente y de calidad se encuentran reconocidos en el artículo 4o., párrafos tercero y cuarto, de la CPEUM.

Desde un punto de vista bioético, el derecho a la alimentación se relaciona con el principio de autonomía, puesto que el consumidor decide qué alimento consumir; sin embargo, para que tal situación suceda, se requiere que sea informado de forma adecuada para obtener el consentimiento de la persona ( $c f r$. García 2013, 69 y Siurana Aparisi 2010, 123 y 124).

De lo anterior, se derivan los derechos como el libre desarrollo de la personalidad, el acceso a la información y la libre elección; es decir, el individuo decide la forma en la cual quiere vivir su vida pues tiene la capacidad de elegir qué es lo que necesita para cumplir con su objetivo, para ello, requiere que sea informado de manera adecuada. ${ }^{3}$ En el caso del derecho a la alimentación se logra mediante el etiquetado, que es el instrumento por el cual se da a conocer el valor nutricional del producto alimenticio. Pero ¿qué sucede cuando el etiquetado es confuso o no logra su objetivo de informar?

Esta situación, tan simple pero relevante, tiene un impacto en el derecho a la alimentación cuando un Estado no establece una regulación en materia de seguridad alimentaria.

3 cfr. rubro: DERECHO AL LIBRE DESARROLLO DE LA PERSONALIDAD. ASPEGTOS QUE COMPRENDE (SCJN 2009, Tesis: P. LXVI/2009), y rubro: DERECHO AL LIBRE DESARROLLO DE LA PERSONALIDAD. SU DIMENSIÓN EXTERNA E INTERNA (SCJN 2019, Tesis: 1a./J. 4/2019 (10a.)). 
Por consiguiente, la pregunta sería ¿qué entiende el Estado mexicano por seguridad alimentaria? Según el artículo 3o., fracción XXVIII, de la Ley de Desarrollo Rural Sustentable es: "El abasto oportuno, suficiente e incluyente de alimentos a la población". Sin embargo, existe una leve pero significativa incompatibilidad entre el derecho humano a la alimentación y la seguridad alimentaria, puesto que la definición debiera contener las características de nutritiva y calidad; en cambio se centra en cantidad mas no calidad del alimento; incluso en la Observación General No. 12 "El derecho a una alimentación adecuada" (artículo 11), en su párrafo 8 señala que el derecho a la alimentación, una de sus partes, comprende "la disponibilidad de alimentos en cantidad y calidad suficientes para satisfacer las necesidades alimentarias de los individuos, sin sustancias nocivas, y aceptables para una cultura determinada [...]".

En el ámbito federal, no existe una legislación en materia de seguridad alimentaria. En cuanto hace al ámbito estatal, sólo ha sido la Ciudad de México quien publicó en la Gaceta Oficial del Distrito Federal, el 17 de septiembre de 2009, la Ley de Seguridad Alimentaria y Nutricional para el Distrito Federal. En lo que concierne a otras entidades federativas, sólo el estado de Oaxaca define el concepto de soberanía y seguridad alimentaria, sin embargo, tal descripción versa en la soberanía alimentaria mas no en la seguridad, conforme a lo estipulado en el artículo 15, fracción XIII de la Ley de Desarrollo Social del estado de Oaxaca, publicado en el Periódico Oficial Extra del 29 de mayo del 2019; mientras las demás reconocen el derecho a la alimentación y es considerado un indicador para medir la pobreza, acorde a las leyes de desarrollo social o las leyes de desarrollo social y humana.

En dicho país, la autoridad competente en materia de etiquetado es la Comisión Federal para la Protección contra Riesgos Sanitarios (Cofepris) el cual es un órgano desconcentrado de la Secretaría de Salud, quien se encarga de regular, controlar, vigilar y fomentar la sanidad en materia de alimentos plaguicidas, etiquetado, entre otros; según lo establecido en el artículo 3o., fracción I, del Reglamento de la Comisión Federal para la Protección contra Riesgos Sanitarios, publicado en el Diario Oficial de la Federación (en adelante DOF), el 13 de abril de 2004.

En 1984, se publicó en el DOF la figura del etiquetado en el artículo 210 de la LGS en donde se estipulaban los datos que debía contener el etiquetado de productos empacados o envasados. Para el 19 de junio de

Esta obra está bajo una Licencia Creative Commons

Atribución-NoComercial-SinDerivar 4.0 Internacional, IIJ-UNAM.

Boletín Mexicano de Derecho Comparado, núm. 158, mayo-agosto de 2020, pp. 763-787. 
2003, este artículo fue reformado y dirigió la regulación de dicho instrumento en las NOM.

La reforma y adición a la LGS, publicado en el DOF el 8 de noviembre de 2019, representó un impacto significativo en materia de sobrepeso, obesidad y de etiquetado de alimentos y bebidas no alcohólicas, ya que se regula la necesidad de prevenir enfermedades y proteger la salud y promover la alimentación nutritiva de la sociedad mediante las dietas, hábitos alimenticios de la población; además de considerar los tratados internacionales de los que México es parte en materia de etiquetado. Por lo que se establece el EFA que indica los límites máximos del valor nutricional, según lo establecido en los artículos 66, primer párrafo; 111, fracción II; 115, fracción VII; 159, fracción V; 212, primer y segundo párrafos, y se adicionan los artículos 2o., con una fracción VIII; 114, con un tercer párrafo; 210, con un segundo párrafo; 212, con tercer y cuarto párrafos, recorriéndose el actual tercer párrafo para pasar a ser quinto párrafo, de la mencionada ley.

Adicionalmente a lo anterior, el artículo 215, fracción VI, de la LGS define al EFA de alimentos y bebidas no alcohólicas, como: "Sistema de información simplificada en el área frontal de exhibición del envase, el cual debe advertir de manera veraz, clara, rápida y simple sobre el contenido que exceda los niveles máximos de contenido energético, azúcares añadidos, grasas saturadas, grasas, sodio y los nutrimentos críticos, ingredientes y las demás que determine la Secretaría [de Salud]".

Derivado de esto, el 27 de marzo de 2020 se publicó en el DOF la reforma a la NOM-051-SCFI/SSA1-2010, Especificaciones generales de etiquetado para alimentos y bebidas no alcohólicas preenvasados-Información comercial y sanitaria (en adelante, NOM-051-SGFI/SSA1-2010 del 2020), con la finalidad de establecer la regulación del EFA en los productos alimenticios, el cual se establece en los puntos 3.47 y 4.5.3. Cabe mencionar que deroga el etiquetado frontal nutricional, señalado en la modificación del 14 de agosto de 2014, en el punto 3.21 y 4.2.9; además de que esta NOM no se aplica a productos a granel, precisado en el punto 1.2, segundo párrafo inciso b.

Una de las problemáticas que tuvo la modificación del 14 de agosto de 2014 sobre dicha NOM, fue que el etiquetado frontal nutrimental no era entendible para el consumidor y no seguía la recomendación internacional emitida por la OMS sobre la disminución del consumo de azúcar, por lo cual la organización no gubernamental "El poder del consumidor" 
promovió el amparo en revisión 240/2018 (WHO 2015 y SCJN 2019, AR 240/2018).

Por lo que, a la modificación NOM-051-SCFI/SSA1-2010 del 2020, se le añade al etiquetado la información nutricional complementaria, es aquí cuando se interpreta la declaración nutrimental del alimento sobre el contenido de azúcares libres, grasas saturadas o trans, y sodio, conforme a los puntos 3.26, 3.38 y 4.5.3.

Para ello, se debe cumplir con las características del sistema de etiquetado frontal: 1) la información nutrimental se muestra mediante sellos y leyendas (por ejemplo, "contiene edulcorantes, no recomendable en niños"; "contiene cafeína- evitar niños") y 2) la información debe ser veraz, directa, clara, sencilla y visible, cuando el alimento contenga un exceso de energía, nutrimentos críticos e ingredientes que dañen la salud humana, de acuerdo a los puntos 3.47, 4.5.3.4, 7.1.3, 7.1.4 de la NOM-051-SCFI/ SSA1-2010 del 2020.

Como se mencionó anteriormente, algunos países (como Chile, Perú, Uruguay, Ecuador $)^{4}$ han establecido el EFA (Rodríguez Osiac y Pizarro Quevedo 2018). Sin embargo, la industria alimentaria se ha pronunciado en contra de este tipo de etiquetado bajo diversos argumentos, los más relevantes han radicado en la estigmatización de los alimentos pues el sello podría enviar un mensaje equivocado que produzca miedo o confusión al consumidor y, por ende, la discriminación del producto alimenticio; además de que no se resuelve el tema de la educación nutricional (Ares, G. et al. 2020; Ministerio de Salud Pública de Chile, 2015).

Aun así, autores como Roger S. Magnusson (2010, 6-7) y, Neha Khandpur et al. (2018, 1-15) argumentan que el etiquetado de semáforo o el frontal de advertencia han tenido un impacto favorable en las decisiones del consumidor debido a que puede identificar los alimentos más saludables, lo cual fomenta la responsabilidad del consumidor sobre su alimentación (también véase Ares, G et al. 2018, 215-225).

Otro argumento que ha empleado la industria alimentaria es la violación a diversos tratados internacionales en materia de comercio, tales como el Acuerdo de Obstáculos Técnicos al Comercio (OTC) de la OMC de 1994, Acuerdos sobre Aspectos de los Derechos de Propiedad Intelec-

\footnotetext{
4 Véase Reglamento Sanitario de Etiquetado de Alimentos Procesados para el Consumo Humano (Acuerdo No. 00004522), Ediciones Legales, 2013.
} 
tual relacionados con el Comercio (ADPIC), Codex alimentarius, Tratado de Libre Comercio con América del Norte (TLCAN) y Tratado entre México, Estados Unidos y Canadá (T-MEG).

Este argumento radica en que el EFA crea un obstáculo innecesario al comercio internacional, se restringe el uso de las marcas con respecto a los personajes que emplean en sus productos (hace alusión a lo establecido en el punto 4.1.5, inciso a de la NOM-051-SCFI/SSA1-2010) y no existe un estándar internacional emitido por el Codex alimentarius sobre el EFA (artículos 2.2, 2.4 del Acuerdo OTC; 15, primer párrafo, del ADPIC; 11.4 del T-MEG).

No obstante, estos tratados le dan sustento al EFA para su implementación bajo los objetivos de: "[...] los imperativos de la seguridad nacional; la prevención de prácticas que puedan inducir a error; la protección de la salud o seguridad humanas [...]", según lo establecido en el artículo 2.2 del Acuerdo OTG.

Ahora bien, este Acuerdo menciona la utilización de las normas internacionales para lograr los objetivos anteriormente mencionados (artículo 2.4 del Acuerdo OTC); sin embargo, las normas emitidas por el Codex alimentarius persiguen la finalidad de: "[... orientar y fomentar] la elaboración y el establecimiento de definiciones y requisitos aplicables a los alimentos para favorecer su armonización y, de esta forma, facilitar el comercio internacional. [Además de que ...] son orientadoras no sustituyen ni son una solución alternativa a la legislación nacional. Las leyes y procedimientos administrativos de cada país contienen disposiciones que es necesario cumplir" (Codex alimentarius, 2020).

Es decir, a pesar de que el EFA restrinja el uso de las marcas en los productos alimenticios, no limita el comercio, sino que lo vuelve más responsable al respetar y proteger los derechos humanos de los consumidores; así como evitar las posibles violaciones a sus derechos, como es el caso del derecho a la salud, tal como lo menciona el artículo 1o. de la CPEUM y los Principios rectores sobre las empresas y los derechos humanos (Naciones Unidades 2011, 3 y 5).

Dicho lo anterior, habría que reflexionar si la modificación a la NOM051-SCFI/SSA1-2010, del 27 de marzo de 2020, contribuye en la solución al problema de salud en materia de obesidad y sobrepeso. Al inicio de este trabajo, se mencionó la falta de estandarización en el etiquetado era 
un factor negativo, aunque queda solventado con el EFA, pero subsiste el problema de origen, la escasa educación nutricional de los consumidores.

Pero, ¿por qué es importante insistir en la educación nutricional, a pesar de tener el EFA, el cual es claro e informa al consumidor sobre el valor nutricional del producto? Esto es equivalente a preguntar ¿por qué enseñarle a un niño de primaria las operaciones básicas (suma, resta, multiplica y dividir), cuando existe la calculadora? O ¿por qué aprender un segundo idioma, cuando existen softwares traductores?

Esto nos conlleva a plantearnos lo siguiente ¿cómo el Estado promueve la responsabilidad nutricional de la población?, ¿existe la educación nutricional? Y desde una perspectiva bioética ¿dónde queda la autonomía del consumidor, cuando la norma prohíbe o facilita su decisión sin enseñarle la forma de cómo balancear los alimentos?

Ahora bien, en la modificación a la NOM, anteriormente mencionada, no considera a los grupos vulnerables, tales como los pueblos indígenas y las personas con discapacidad auditiva o visual. Este aspecto resulta relevante puesto que desde el 2015 México se comprometió a cumplir con los objetivos para el desarrollo sustentable de la Agenda 2030 y, justamente, la inclusión y la educación son dos objetivos fundamentales para lograr el mejoramiento de la calidad de vida, la igualdad de oportunidades y proteger los derechos humanos de estos grupos (cfr. López Ricalde et al. 2005 y United Nations 2017, A/RES/71/313).

De tal manera que el derecho a la educación en materia de nutrición es un factor importante para la toma de decisiones sobre la adquisición y consumo de un alimento. Además de que forma parte de los principios para las buenas prácticas comerciales en donde: "Las empresas deben elaborar, según proceda, programas y mecanismos para ayudar a los consumidores a adquirir los conocimientos y competencias necesarios para comprender los riesgos, incluidos los riesgos financieros, tomar decisiones bien fundadas y acceder a servicios competentes y profesionales de asesoramiento y asistencia, prestados preferiblemente por terceros independientes, cuando sea necesario" (según el párrafo 11, inciso d, de las Directrices de la Naciones Unidas para la Protección del Consumidor; Naciones Unidades 2011).

Dicho esto, la industria alimenticia desde una perspectiva de derechos humanos, está obligada a tratar con igualdad a sus consumidores sin discriminación alguna, con la finalidad de respetar su autonomía y libertad 
para decidir conforme a la información brindada de sus productos sin que medie obstáculo ( $c f r$. párrafo 11 de las Directrices de la Naciones Unidas para la Protección del Consumidor). Adicionalmente, la Observación General No. 12 "El derecho a una alimentación adecuada" (artículo 11), en su párrafo 15, señala que el derecho a la alimentación debe considerar a las minorías y prevenir cualquier tipo de discriminación. Asimismo, existe la obligación por parte del Estado de respetar, proteger y realizar por medio de la adopción de medidas el acceso a una alimentación adecuada (ONU-Consejo Económico y Social. Aplicación del Pacto Internacional de los Derechos Económicos, Sociales y Culturales 1999).

\section{EL USO DE LAS TIG EN EL ETIQUETADO DE ALIMENTOS}

En la sección anterior, se analizó el contexto por el cual se modifica la NOM-051-SCFI/SSA1-2010 y las posturas al establecer el EFA como un código en común, en donde la mayor parte de los consumidores son informados y comprender el valor nutricional del producto alimenticio, con la finalidad de decidir por alimentos más saludables. Sin embargo, queda pendiente la educación nutricional del consumidor.

Según la Organización de las Naciones Unidas para la Alimentación (por sus siglas en ingles FAO), se entiende por educación nutricional como "[...] la difusión de información acerca de los alimentos y sus nutrientes, $[\ldots$ así como ... $]$ proporciona $[\mathrm{r}]$ las herramientas para saber qué hacer y cómo actuar para mejorar la nutrición" (FAO 2020).

No obstante, como se expuso en la sección anterior, esta educación debe ser incluyente y considerar a grupos vulnerables como los pueblos indígenas y las personas con discapacidad visual y auditiva.

Para ello, una herramienta que puede contribuir en la lucha contra la obesidad y la diabetes mediante la educación nutricional es el uso de las TIC, el cual es definido por Juan Cristóbal Cobo Romaní como:

Dispositivos tecnológicos (hardware y software) que permiten editar, producir, almacenar, intercambiar y transmitir datos entre diferentes sistemas de información que cuentan con protocolos comunes. Estas aplicaciones, que integran medios de informática, telecomunicaciones y redes, posibilitan tanto la comunicación y colaboración interpersonal (persona a persona) como la multidireccional (uno a muchos o muchos a muchos). Estas herramien- 
tas desempeñan un papel sustantivo en la generación, intercambio, difusión, gestión y acceso al conocimiento (Cobo Romaní 2009, 312).

Esta definición señala un amplio panorama, el cual las TIC pueden ser utilizadas en cualquier materia (como en el etiquetado y la educación nutricional), ya que puede incorporarse tecnologías como la realidad aumentada, cómputo en la nube, internet de las cosas, entre otras. Especialmente, las TIC pueden contribuir a aminorar la desigualdad social (INEHRM-GNDH 2015). Cabe mencionar que el artículo 6o. de la CPEUM consagra el derecho al acceso y uso de las tecnologías de la información y la comunicación.

Dicho lo anterior, se propone el uso del código QR y la realidad aumentada, ya que ambas tecnologías pueden ser empleadas para brindarle una información más clara y precisa al consumidor; además de otorgar un aprendizaje sobre educación nutricional.

A continuación, se explica cómo pueden emplearse ambas tecnologías para tales fines:

\section{El código QR (quick response code)}

Este código de barras bidimensional permite almacenar información y otorga la posibilidad de acceder a sitios web u otros recursos digitales; asimismo, puede ser leído mediante cualquier dispositivo electrónico que tenga la tecnología para su procesamiento (Carrillo-Larco y H. Curioso 2013 y Liu et al. 2012). Además, tal tecnología se encuentra regulada bajo el estándar internacional ISO/IEC 18004 Information technology-Automatic identification and data capture techniques-Bar code symbology-QR Code, First edition 2000-06-15. Es menester mencionar que en las NOM no existe una regulación para la aplicación del código QR en los productos alimenticios.

En otro aspecto, el código $\mathrm{QR}$ tiene una capacidad acorde a su formato: numérico (7089 caracteres), alfanumérico (4296 caracteres), binario (2953 bytes) y kanji/kana (1817 caracteres) (Padrón Godínez et al. 2020, 3). La creación de dicho código es mediante un programa determinado como $Q R$ code generator o código QR de Beaconstac.

Con respecto al tema del etiquetado de alimentos, el estudio realizado por Dalia Stern et al. (anteriormente mencionado) analizaron que el etiquetado frontal de las GDA tenía sus limitantes relativas al consumo

Esta obra está bajo una Licencia Creative Commons

Atribución-NoComercial-SinDerivar 4.0 Internacional, IIJ-UNAM.

Boletín Mexicano de Derecho Comparado, núm. 158, mayo-agosto de 2020, pp. 763-787. 
máximo diario ya que se estandarizan su valor acorde a un tipo de persona y su condición física, es decir, no se discrimina si es niño o adulto, ni su género (2011, 13 y 14).

Por tal motivo, emplear el código $\mathrm{QR}$ en el etiquetado brindaría al consumidor un mayor entendimiento sobre la información nutricional del producto, pues se ofrecerían datos más detallados y entendibles para los consumidores, lo cual da lugar al consentimiento informado para la adquisición y consumo de un producto bajo la finalidad de llevar una dieta balanceada y saludable.

Por último, el código $\mathrm{QR}$ puede ser empleado de diversas maneras, según la finalidad que se persiga, tales como: transmitir la información en materia de salud (Lin et al. 2012 y Carrillo-Larco y Curioso Walter 2013), enseñar alguna asignatura escolar (Bonifácio 2012, 71 y 72), innovar la industria periodística impresa al redireccionar al lector a sitios web para ver videos u otro recurso (Meneses Fernández et al. 2014), entre otras; puesto que en este código se pueden almacenar y codificar diversidad de recursos multimedia.

Es así como, en materia de etiquetado y educación nutricional, este código puede ser utilizado como un instrumento viable para brindar una información más detallada sobre el valor nutricional del alimento.

Adicionalmente, es necesario mencionar que su aplicación podría aplicar no sólo a los alimentos y bebidas no alcohólicas preenvasados sino también, a los productos a granel e incluso para lograr la inclusión de los consumidores de los grupos vulnerables como los pueblos indígenas y las personas con discapacidad.

\section{La realidad aumentada $(R A)$}

Esta tecnología versa en sobreponer "[...] a una imagen real obtenida a través de una pantalla imágenes, modelos $3 \mathrm{D}$ u otro tipo de informaciones generados por ordenador" (Prendes Espinosa 2015, 187).

Dicho en otras palabras, se basa en que, a través de un objeto físico, el individuo pueda interactuar en un entorno donde lo real y lo virtual se combinan dentro de tiempo y momento determinado, es decir, mediante un ordenador se elaboran elementos virtuales en 3D que se sobreponen en el entorno real, apoyados mediante un display, ya sea con gafas (Head 
Mounted Displays, HMDs) o por medio de un dispositivo móvil, entre otros equipos electrónicos adaptados a esta tecnología (Ruiz Torres 2011, 215 y 216; Billinghurst et al. 2001, 745 y 746).

La RA tiene aplicación para distintos ámbitos, como el educativo. Por lo que, tal herramienta puede emplearse para fomentar la educación nutricional del consumidor. Asimismo, para la elaboración de esta tecnología se requiere de programas específicos como Adobe Aero, Mergecube, Creator de Aumentaty, entre otras; lo cual permite innovar la forma de enseñanzaaprendizaje.

$\mathrm{Al}$ revisar la literatura enfocada en la RA aplicada al ámbito educativo, se muestra cómo ha sido empleada esta tecnología para tales fines. Algunos ejemplos son:

a) MagicBook elaborado por el Human Interface Technology Laboratory de la Universidad de Washington, el cual es un libro físico que contiene marcadores (conocidos como interfaces tangibles de usuario) que, al ser visualizados mediante un equipo de realidad virtual, la persona puede interactuar con el contenido de forma natural (Billinghurst et al. 2001, 745-753).

b) Piano virtual y partitura virtual: el primero, es una herramienta que emula los sonidos de un piano mediante la lectura del posicionamiento de las manos; mientras el segundo, la herramienta da oportunidad de crear melodías con base en el empleo de pentagramas y notas musicales (Peula et al. 2007).

Además de estos ejemplos se añade el uso de la RA en los museos (Ruiz Torres 2011).

De esta forma, el uso de las TIC en materia de etiquetado, ofrece la oportunidad para fomentar la educación nutricional del consumidor y no sólo un sello que le advierta, sino que sea consiente de cómo utilizar esa información para saber de qué forma mejorar su nutrición y sus hábitos alimenticios.

De igual manera, su uso coadyuvaría en la encomienda que tienen la Secretaría de Educación Pública y la Secretaría de Salud, en colaboración con el sector social y privado, en fomentar "[...] programas de orientación y educación para una alimentación saludable y nutritiva que mejore la ca- 
lidad de vida de las niñas [..., niños y adolescentes]", según lo establecido en el artículo 41 de la Ley General de Educación. ${ }^{5}$

\section{LOS OBSTÁCULOS DEL ETIQUETADO DIGITAL}

Hasta el momento hemos abordado que el uso de las TIC en materia de etiquetados podría contribuir en el fomento de la educación nutricional, lo cual puede ser novedoso; sin embargo, se debe de considerar sus limitaciones en cuanto a su viabilidad, factibilidad y aplicabilidad, por lo cual, en este trabajo se consideran las siguientes:

\section{Analfabetismo}

En México, la población analfabeta de 15 y más años de edad representaba el 5.5\%, es decir, 4,749,057 personas, según los datos de la Encuesta Intercensal de 2015 del Instituto Nacional de Estadística y Geografia (en adelante INEGI 2015). En cuanto hace a los hablantes de lengua indígena, de ese mismo año, representaban el 23.0\%, lo que a nivel nacional era equivalente al 5.5\%; sin embargo, para ese año el mayor índice de analfabetas residía en las personas de 65 años en adelante (INEGI 2015).

Con respecto al analfabetismo digital, según la Encuesta Nacional sobre Disponibilidad y Uso de Tecnologías de la Información en los Hogares (ENDUTIH) de 2019, reflejó lo siguiente:

En los hogares se cuenta con el equipamiento de tecnología de información y comunicaciones tales como computadora (44.3\%); conexión a Internet (56.4\%); telefonía (92.5\%), entre otros.

No obstante, las viviendas puede que no dispongan con un equipo de cómputo por diversas razones, algunas de ellas son: falta de recursos económicos (53.3\%); no les interesa (20.9\%); no saben usarla (16.5\%); utilizan un smartphone u otro dispositivo para conectarse a Internet (5.6\%); entre otras. (INEGI 2019a).

5 Este artículo se relaciona con las Directrices de la Naciones Unidas para la Protección del Consumidor en la letra G. Programas de educación e información, a partir del párrafo 42 al 48. 


\section{Acceso a la tecnología}

Cada día, la innovación tecnológica se adapta mejor a las necesidades sociales; a pesar de todo, hay que considerar que algunas personas no tienen acceso a estas innovaciones y conservan tecnología obsoleta, como es el caso de los dispositivos móviles, lo cual podría dificultar su transición y acceso a la lectura de un etiquetado digital con base al código $\mathrm{QR}$ o a la RA ya que tales dispositivos serían incapaces de leer las interfaces tangibles de usuario.

Algunos datos proporcionados en la ENDUTIH de 2019, mostraron que $35.5 \%$ de la población no disponía de un teléfono móvil por falta de recursos; mientras $1.6 \%$ declaró que no hay servicio en su localidad ya sea por falta de infraestructura o por cobertura en red (INEGI 2019a).

\section{Cobertura de Internet}

Las TIC (el código QR y la RA) requieren el uso del Internet; sin embargo, en México, la cobertura no está al 100\%, lo cual es un obstáculo para que ciertas comunidades queden excluidas de una instauración de etiquetado digital.

En México, el Instituto Federal de Telecomunicaciones (IFT) es el órgano autónomo encargado de “[...] la regulación, promoción y supervisión del uso, aprovechamiento y explotación del espectro radioeléctrico, los recursos orbitales, los servicios satelitales, las redes públicas de telecomunicaciones y la prestación de los servicios de radiodifusión y de telecomunicaciones, así como del acceso a la infraestructura activa y pasiva y otros insumos esenciales [...]”, según lo establecido en el artículo 7o. de la Ley Federal de Telecomunicaciones y Radiodifusión, publicado en el DOF el 14 de julio de 2014 y reformado el 24 de enero de 2020.

Conforme a la ENDUTIH de 2019, algunas de las causas por la cual las residencias no dispongan de la conexión a Internet, tales como: falta de recursos económicos (50.7\%); no les interesa o no lo necesitan (24.4\%); no hay proveedores o infraestructura en su localidad (15.9\%); entre otros aspectos (INEGI 2019a).

No obstante, en 2018 el IFT publicó el diagnóstico titulado "Cobertura del servicio móvil en pueblos indígenas, con base en información 
proporcionada por los concesionarios en el año 2018”, cuyos hallazgos fueron:

1) El 88\% de población indígena cuenta con cobertura móvil (sea 2G, $3 \mathrm{G} \mathrm{o} 4 \mathrm{G})$.

2) Sólo 16 pueblos indígenas de los 66 identificados en México, contaban con una cobertura de servicios móvil al 100\% (sea 2G, 3G o $4 \mathrm{G})$.

3) El 82\% de la población indígena contaba con cobertura 3G (IFT $2018,8)$.

\section{Etiquetado incluyente.}

Para este aspecto, se consideran dos grupos vulnerables: los pueblos indígenas y las personas con discapacidad visual o auditiva; dado al compromiso adquirido en el 2015 con la Agenda 2030 para el desarrollo sustentable.

Bajo ese mismo tenor y conforme a lo desarrollado en este trabajo, recordemos que el EFA no soluciona el problema de la educación nutricional, sino que facilita la comprensión de la información nutricional sobre un producto alimenticio al establecer un código común para la mayor parte de la población. Sin embargo, no se considera que el Estado mexicano es pluricultural y que existen personas con alguna discapacidad; por ende, se podría vulnerar la autonomía de estos grupos vulnerables al no ser informados correctamente y, con ello, su dignidad humana al ser excluidos por no adoptar las medidas necesarias para garantizar su derecho a la salud y, a la alimentación nutritiva y de calidad.

Por tal motivo, el objetivo 4o. del desarrollo sustentable, bajo su indicador 4.5, establece el aseguramiento del acceso igualitario para una educación inclusiva, lo cual involucra a los pueblos indígenas y a personas con discapacidad (Sustainable Development Goal 2017, A/RES/71/313).

No obstante, incluir a ambos grupos vulnerables representa un reto para la industria de alimentos por lo siguiente:

1) La obra del Instituto Nacional de Lenguas Indígenas titulado Catálogo de las lenguas indígenas nacionales: variantes lingüísticas de México con sus autodenominaciones y referencias geoestadísticas, publicado en el DOF 
el 14 de enero de 2008, expone la variedad lingüística indoamericana en México concentrada en 11 familias (por ejemplo, Álgica, Yuto-nahua, Cochimí-yumana, Seri, Oto-mangue, Maya), que a su vez tiene 68 agrupaciones lingüísticas, y de las cuales se derivan 364 variedades lingüísticas.

2) Con respecto a las personas con discapacidad, en México, existen 7.7 millones de personas de 5 años y más que son consideradas como población con discapacidad, es decir, 6.7\%, según la Encuesta Nacional de la Dinámica Demográfica (ENADID) 2018. Específicamente, esta encuesta expuso que $39.6 \%$ de la población tiene una discapacidad visual (aunque utilice lentes); mientras que la auditiva equivale a $24.7 \%$ (aunque utilice aparato auditivo) (INEGI 2019b).

Ambos grupos vulnerables son la minoría de la población mexicana; sin embargo, al ser sujetos de derechos humanos se les debe de proteger, promover y garantizar sus derechos sin ninguna discriminación. Por tal motivo, en materia de etiquetado de alimentos se requiere ajustes razonables para adaptarlos acorde a sus necesidades, según lo establecido en el artículo 2o. de la Convención sobre los Derechos de las Personas con Discapacidad. Por ejemplo, en el caso de las personas con discapacidad visual podría emplearse el código QR de voz; en cuanto hace a la discapacidad auditiva, podría ser un código $\mathrm{QR}$ de video en donde se maneje el lenguaje de señas para comunicar el contenido del alimento. Esto mismo, podría aplicar con las personas indígenas. De esta forma, se conseguiría un trato digno para esta población.

\section{CONCLUSión}

La implementación de un etiquetado digital en materia de alimento podría contribuir en la educación nutricional del consumidor y hacer más consciente de lo que consume; así como darle la posibilidad de poder llevar una dieta balaceada y nutritiva, puesto que del derecho a la alimentación dependen los demás derechos humanos ( $c f r$. Naciones Unidas 1999).

Asimismo, este tipo de etiquetado podría contribuir en aminorar la desigualdad social e incluir a los grupos vulnerables. De esta forma, las 
TIC se convierten en un aliado para fomentar la educación nutricional del consumidor; mediante la colaboración del Estado, las empresas y el propio consumidor, y contribuir en la lucha contra la obesidad y la diabetes.

Por último, es importante comprender que se pueden desarrollar las mejores políticas públicas, aumentar el impuesto a las comidas chatarra, establecer el EFA o el etiquetado por semáforo, entre otras estrategias, pero si no se comienza a promover y fomentar la educación nutricional para cambiar hábitos alimenticios, el problema podría persistir durante años.

\section{BIBLIOGRAFÍA}

ARAYA, Eric. 2017. Abecé de redacción. España. Océano exprés.

AREs, Gastón et al. 2020. "Argumentos de la industria alimentaria en contra del etiquetado frontal de advertencias nutricionales en Uruguay", Rev Panam Salud Publica 44. https://doi.org/10.26633/RPSP.2020.20.

Billinghurst, Mark et al. 2001. "The MagicBook: A transitional AR interface". Elsevier Computers \& Graphics, 25 (5): 745-753. https://doi. org/10.1016/S0097-8493(01)00117-0.

BonifÁcio, V. 2012. "Ensinar Química com telemóveis inteligentes: a tabela periódica em código QR. Química”. Sociedade Portuguesa de Química, serie II, 127,71 y 72 .

Carrillo-Larco, Rodrigo M. y Curioso, Walter H. 2013. "Oportunidades del código QR para diseminar información en salud [carta]". Revista Peruana de Medicina Experimental y Salud Publica. 30(2): 362 y 363.

Cobo Romaní, Juan Cristóbal. 2009. "El concepto de tecnologías de la información. Benchmarking sobre las definiciones de las TIC en la sociedad del conocimiento". Zer. 14(27), 295-318.

Codex Alimentarius. 2020. "Acerca del Codex". http://wrerw:fao.org/faowho-codexalimentarius/about-codex/es/ (última consulta 11 de septiembre de 2020).

Cowburn, Gill y STOCKley, Lynn. 2005. "Consumer understanding and use of nutrition labelling: a systematic review". Public Health Nutrition, 8(1): 21-28. DOI: 10.1079/PHN2004666. 
DíAZ, Adrián Alberto et al. 2017. "Etiquetado de alimentos en Ecuador: implementación, resultados y acciones pendientes". Revista Panam Salud Pública, 41, 1-8.

FAO-Programa Especial para la Seguridad Alimentaria (PESA), Centroamérica. 2011. Seguridad alimentaria y nutricional. Conceptos básicos, 3a. ed., FAO. http://wrwe.fao.org/3/a-at772s.pdf (última consulta 29 de diciembre de 2019).

FAO. 2019. "Etiquetado frontal de advertencia, un paso urgente para enfrentar epidemia de sobrepeso y obesidad en México". FAO en México, 30 de septiembre de 2019. http://wrere.fao.org/mexico/noticias/detailevents/es/c/1235765/(última consulta 14 de enero de 2020).

FAO. 2011. La seguridad alimentaria: información para la toma de decisiones. Guía práctica. Programa CE-FAO. http://wrere.fao.org/3/al936s/al936s00.pdf (última consulta 20 de agosto 2020).

FAO. 2019. "Etiquetado frontal de advertencia, un paso urgentepara enfrentar epidemia de sobrepeso y obesidad en México". FAO en México. http:// wrere.fao.org/mexico/noticias/detail-events/es/c/1235765/ (última consulta 14 de enero de 2020).

Ferrajoli, Luigi. 2011. Poderes salvajes. La crisis de la democracia constitucional, trad. Andrés Ibánez, 2a. ed., España, Trotta.

García, José Juan. 2013. "Bioética personalista y bioética principialista. Perspectivas". Cuadernos de bioética, 24(1), 67-76.

GASTÓn, Ares et al. 2018. "Comparative performance of three interpretative front-of-pack nutrition labelling schemes: Insights for policy making". Food Quality and Preference, 68, 215-225.

IFT. 2018. Cobertura del servicio móvil en pueblos indigenas, con base en información proporcionada por los concesionarios en el año 2018. México. http://wrere.ift. org.mx/sites/default/files/reporte-coberturapueblosindigenas_finalpublicar_2_0. $p d f$ (última consulta 21 de agosto de 2020).

INEGI. 2015. Encuesta Intercensal 2015. https://wrewe.inegi.org.mx/programas/ intercensal/2015/(última consulta 20 de enero 2020).

INEGI. 2019a. Encuesta Nacional sobre Disponibilidad y Uso de Tecnologías de la Información en los Hogares (ENDUTIH), 2019. https://wrwe.inegi.org.mx/ programas/dutih/2019/default.html\#Documentacion (última consulta 20 de enero de 2020).

INEGI. 2019b. Estadísticas a propósito del día internacional de las personas con discapacidad (3 de diciembre). Datos Nacionales Comunicado de Pren-

Esta obra está bajo una Licencia Creative Commons

Atribución-NoComercial-SinDerivar 4.0 Internacional, IIJ-UNAM.

Boletín Mexicano de Derecho Comparado, núm. 158, mayo-agosto de 2020, pp. 763-787. 
sa, núm. 638/19: 1-11. https://wrere.inegi.org.mx/contenidos/saladeprensa/ aproposito/2019/Discapacidad2019_Nal.pdf(última consulta 20 de agosto de 2020).

Instituto Nacional de Estudios Históricos de las Revoluciones de México (INEHRM) y la Comisión Nacional de los Derechos Humanos (GNDH). 2015. Derecho de acceso y uso de las tecnologías de la información y la comunicación. Colección INEHRM, Centenario de la Constitución de 1917. México. UNAM, Instituo de Investigaciones Jurídicas.

Instituto Nacional de Salud Pública y UNICEF México. 2016. Encuesta Nacional de Niños, Niñas y Mujeres 2015-Encuesta de Indicadores Múltiples por Conglomerados 2015, Informe Final. México. Instituto Nacional de Salud Pública y UNICEF México.

KHANDPUR, Neha et al. 2018. "Are Front-of-Package Warning Labels More Effective at Communicating Nutrition Information than TrafficLight Labels? A Randomized Controlled Experiment in a Brazilian Sample". Nutrients, 10. 688: 1-15. DOI:10.3390/nu10060688.

LIN C.H. et al. 2012. "The feasibility of QR-code prescription in Taiwan". Fournal of Clinical Pharmacy and Therapeutics, 37: 643-646. DOI: 10.1111/j.1365-2710.2012.01358.x.

LIU, Chang, et al. 2012. "DNA barcode goes two-dimensions: DNA QR code web server", PloS one, 7 (5). e 35146: 1-7. https://doi.org/10.1371/ journal.pone.0035146.

LóPEz Ricalde, Carlos David et al. 2005. "Desarrollo sustentable o sostenible: una definición conceptual”. Horizonte Sanitario, 4(2), 1-6.

Magnusson, Roger S. 2010. "Obesity prevention and personal responsibility: the case of front-of-pack food labelling in Australia". BMC Public Health, 10:662: 1-12. DOI:10.1186/1471-2458-10-662.

MAZHAR, Siraj. 2011. "Protection and advancement of Human Rights in developing countries: Luxuries or Necessities?". Human Affairs, Springer, 21(304). https://doi.org/10.2478/s13374-011-0031-7.

Meneses Fernández, María Dolores et al. 2014. "Audiovisualización del papel. Usos del código QR para innovar en la industria periodística impresa". INNOVAR. Revista de Ciencias Administrativas y Sociales, 24 (1), 67-80.

Ministerio de Salud Pública de Chile. 2015. "Consolidado de respuestas a observaciones recibidas durante consulta pública nacional e internacional sobre propuesta de modificación del Decreto Supremo N977/96”. 
Reglamento Sanitario de los Alimentos. Ministerio de Salud de Chile, para la ejecución de la Ley $\mathrm{N}^{\circ}$ 20.606, sobre composición nutricional de los alimentos y su publicidad, Chile, Ministerio de Salud.

Montes de Oca BARrera, Laura Beatriz. 2019. Comida chatarra. Entre la gobernanza regulatoria y la simulación. México. UNAM, Instituto de Investigaciones Sociales.

Naciones Unidas. 2000. Observación General No. 14, El derecho al disfrute del más alto nivel posible de salud. Ginebra. Consejo Económico y Social, E/C.12/2000/4.

Naciones Unidas. 1999. Observación General No. 12 El derecho a una alimentación adecuada (artículo 11). Ginebra. Consejo Económico y Social. E/C.12/1999/5.

NACiOnes UnidAS. 2011. Principios rectores sobre las empresas y los derechos humanos. Nueva York y Ginebra. https://wrere.ohchr.org/documents/publi cations/guidingprinciplesbusinessh_sp.pdf (última consulta 20 de enero de 2020).

OPS-OMS. 2012. "Estrategia para la prevención y el control de las enfermedades no transmisibles, 2012-2025". 28.a Conferencia Sanitaria Panamericana, 64.a Sesión del Comité Regional. CSP28/9. Rev. 1 (Esp.). Washington, D. C.

Organización de las Naciones Unidas para la Alimentación (por sus siglas en inglés FAO). 2020. "Educación Alimentaria y Nutricional". Nutrición. http://wrwrefao.org/nutrition/educacion-nutricional/es/(última consulta 10 de septiembre de 2020).

PADRÓn GODÍnEZ, Alejandro et al. 2020. "Confidencialidad de datos mediante el grabado de códigos QR cifrados: ID-óptico". Revista de $I+D$ Tecnológico, 16 (2): 1-9. https://doi.org/10.33412/idt.v16.2.2832.

PAz González, Issac de. 2017. Constitucionalismo y justiciabilidad de los derechos sociales. Estudio comparado internacional y leading cases a través del juicio de amparo en México. México. Porrúa.

PEULA, J.M. et al. 2007. "Realidad aumentada aplicada a herramientas didácticas musicales". XXII Simposium Nacional Unión Científica Internacional de Radio (URSI 2007). España.

Prendes EsPinosa, Carlos. 2015. "Realidad aumentada y educación: análisis de experiencias prácticas". Revista de Medios y Educación, 46, 187 203. http://dx.doi.org/10.12795/pixelbit.2015.i46.12. 
Rodríguez Osiac, Lorena y Pizarro Quevedo, Tito. 2018. "Ley de Etiquetado y Publicidad de Alimentos: Chile innovando en nutrición pública una vez más". Revista Chilena de Pediatría, 89(5): 579-581. http:// dx.doi.org/10.4067/S0370-41062018005000806.

RuIz TORRES, David. 2011. "Realidad aumentada, educación y museos". Revista Icono14. 9(2), 212-226.

SCJN. "Reformas constitucionales en materia de amparo y derechos humanos publicadas en junio de 2011 (Relación de tratados internacionales de los que el Estado mexicano es parte en los que se reconocen derechos humanos)". http://wrwe.internet2.scjn.gob.mx/red/constitucion/(última consulta 12 de julio de 2019).

SIURANA APARISI, Juan Carlos. 2010. "Los principios de la bioética y el surgimiento de una bioética intercultural". Veritas, núm. 22, 121-157. http://dx.doi.org/10.4067/S0718-92732010000100006.

STERn, Dalia, et al. 2011. "Revisión del etiquetado frontal: análisis de las guías diarias de alimentación (GDA) y su comprensión por estudiantes de nutrición en México". Cuernavaca. Instituto Nacional de Salud Pública.

Sustainable Development Goal. 2017. Resolution adopted by the General Assembly on Work of the Statistical Commission pertaining to the 2030 Agenda for Sustainable Development (A/RES/71/313). https://unstats.un.org/sdgs/indi cators/Global\%20Indicator\%20Framework_A.RES.71.313\%20Annex.Spa nish.pdf (última consulta 02 de febrero de 2020).

UNCTAD. 2016. Directrices de la Naciones Unidas para la Protección del Consumidor. Nueva York y Ginebra. Naciones Unidas.

United Nations. 2017. Work of the Statistical Commission pertaining to the 2030 Agenda for Sustainable Development (A/RES/71/313), resolution adopted by the General Assembly. https://cea.cepal.org/9/en/documents/work-statistical-com mission-pertaining-2030-agenda-sustainable-development (última consulta 21 de agosto de 2020).

UPENDRA, Baxi. 2007. Human Rights in a Post Human World: Critical Essays. Oxford University Press. Dehli.

WHO. 2015. Guideline: Sugars intake for adults and children. Geneva. World Health Organization. 